\title{
NUMERICAL AND EXPERIMENTAL ANALYSIS OF A FRICTIONLESS RECEDING CONTACT BETWEEN CYLINDRICAL INDENTER, LAYER AND SUBSTRATE
}

\begin{abstract}
Summary
This paper investigates the behaviour of a receding contact when a cylindrical indenter presses an unbonded layer resting on a substrate. The problem is analysed by using FEM within the scope of the linear theory of elasticity and under the assumption of plane strain. This paper presents new and original results in the analysis of influence of load intensity and indenter geometry on the contact parameters. In addition, in the investigation into material properties a reference analysis was carried out for the case of material similarity between all three bodies, and material properties were subsequently varied for each body. This class of problems shows nonlinear behaviour, with both contact pressure distributions and contact half-widths found to depend nonlinearly on the applied load. The experimental analysis was carried out by employing the digital image correlation method and the ARAMIS 4M system was used. The obtained measurement results show good agreement with the numerical results.
\end{abstract}

Key words: $\quad$ receding contact problem, unbonded layer, FEM, digital image correlation

\section{Introduction}

In a great majority of contact problems the area of the contact surface increases with deformation as the intensity of the applied load increases. However, a separate class of conforming contact problems deviates from this behaviour; in these cases the contact area shrinks with the application of load and such a contact is referred to as a receding contact. In a more precise definition given by $\mathrm{K}$. L. Johnson, a receding contact is one where the loaded contact area is completely contained within the unloaded contact area [1]. Receding contact typically occurs in problems of unbonded layers pressed against a substrate. This type of structural problem is mainly encountered in foundations, pavements and railways, but it has also been studied in connection to tilting pad bearings and resistance spot welding problems.

The receding phenomenon for the layer-substrate type of problems was predicted quite early, in 1903 by Filon [2], who investigated the behaviour of beams under various types of bending. Among those he investigated the problem of an elastic block of a rectangular crosssection pressed into a smooth rigid plane by a concentrated load acting along a line of its upper surface transverse to its length, with the implicit assumption that the length of the block is sufficiently greater than the block height $h$. His analysis predicted the occurrence of tension, 
instead of pressure, at a distance approximately $1.35 \mathrm{~h}$ away from the point of the application of the concentrated load. This finding pointed to the conclusion that separation between the two contacting surfaces must take place when load is applied.

Few experimental measurements were made over the course of an entire century. Filon reported in [2] that experiments with blocks made of rubber showed that the phenomenon of receding contact is real; although he did not refer to the fact that rubber is not an isotropic material, the results for contact half-width were of the order of his prediction of $1.35 \mathrm{~h}$. Some experimental studies on this subject published in later decades known to the authors are the photoelastic studies by Frocht in 1943, by Cocker and Filon in 1957, in which Filon's earlier results regarding contact width were confirmed to a reasonable degree, and by Durelli, Parks and Nørgård in 1973 [3]. The results given in [3] were interesting at the time to the extent that they revealed the inadequacy of earlier theoretical results, especially for the estimation of maximal contact pressure between the layer and the substrate, which proved to be significantly lower in reality than the values predicted by existing theoretical solutions.

This class of contact problems was thoroughly investigated in numerous analytical and numerical studies by many researchers over the last few decades. Weitsman [4] addressed the problem in a study of an elastic layer indented into a half-space by a point load, where the layer was modelled in accordance with the plate theory - this approach produced quite incorrect results, whose inaccuracy deteriorated by shrinking the contact area into a single point as the half-space would approach the properties of a perfectly rigid solid. Weitsman also analysed the plane case in [5] by treating the layer as an infinite beam resting on a half-space, but also failed to obtain good results. Keer, Dundurs and Tsai treated the problem in [6] by employing 2D elasticity theory equations for both the layer and the half-space and obtained results for the case of a symmetric distribution of load around the centre of the loading interval and then generalized their results in [7] to the cases when load distribution is not symmetrical; their result regarding the contact area width of approximately $0.85 h$ is well below Filon's value. Gladwell also investigated the problem of an infinite beam pressed by a concentrated force in [8] and for small $h$ obtained results in very good agreement with [6]. In [9], Ratwani and Erdogan extend the problem by introducing a rigid cylindrical indenter into the model (instead of an assumed pressure distribution), and in [10], Civelek and Erdogan for the first time take the elasticity of the indenter into account, however, they do not analyse explicitly the influence of load intensity on the contact width.

Among the researchers who tackled the problem using numerical methods, Garrido, Foces and Paris employed the boundary element method (BEM) in [11] under the assumption of small displacements, while Garrido and Lorenzana in [12] extended the results from [11] to the case of large displacements; here, the indenter was also replaced by an assumed pressure distribution. Garrido and Lorenzana obtained good agreement with analytical solutions from [6] for the case of small displacements, and rather noticeable difference for maximal contact pressures (17-18\%) and contact width (cca 5\%) for the case of large displacements. Kauzlarich and Greenwood made a 3D FEM analysis in [13] to investigate the case of a centrally loaded plate on both rigid and elastic base, and for the width of the contact area they obtained values in good agreement with [6], i.e. significantly lower values in comparison to Filon's solution.

In later studies, Comez, Birinci and Erdol examined in [14] the case of two elastic layers resting one on top of another indented by a rigid stamp, with the bottom layer bonded to a rigid support; their approach allows for any profile of the indenter that can be described mathematically in closed form. The case of anisotropy was investigated in papers [15]-[17], with particular reference to the results in [16] and [17], where the layer was modelled as a functionally graded material (FGM). Ahn and Barber investigated the case of frictional contact [18] under cyclic loading, but they used a uniformly distributed pressure of constant width. 
Comez took friction on both contacting surfaces into account in [19] but somewhat simplified the problem by a rigid indenter. Some of more recent studies available in literature can be found in [20]-[23]. In [20], Adibelli, Comez and Erdol investigated a receding contact of a coated layer, with emphasis on the influence of the coating to contact parameters. In [21], Öner, Yaylaci and Birinci compared analytical solutions to a FEM solution for the case of two infinite layers supported by a Winkler foundation, obtaining nearly perfect agreement between the two solutions; in [22], Birinci, Adiyaman et al. compared analytical and FEM solutions to the same problem, this time taking body forces into account as well. In [23], Öner, Yaylaci and Birinci compared analytical solutions and FEM in a comprehensive analysis of two unbonded layers resting on an elastic half-space, but with the indenter taken to be rigid.

In this study, we consider the case of an elastic cylindrical indenter pressing an unbonded layer resting on an elastic support. The problem is modelled under the plane strain assumption and is assumed to be frictionless, implying that only compressive tractions can be transmitted on the contacting surfaces. All bodies in contact (indenter, layer and substrate) are assumed to be elastic and isotropic, with the elasticity of the indenter leading to continuous change of the contact width as load is increased. In Chapter 2, a brief description of the numerical model and the Femap software in contact modelling is outlined in the first part, and numerical results are presented and discussed in the second part. In Chapter 3, a description of the experimental setup and the procedure of the experiment preparation is given, followed by the experimental results obtained by digital image correlation method. Finally, in Chapter 4 , a short discussion of the obtained results is presented and complemented by an outlook to future research.

\section{Finite element analysis}

\subsection{Numerical model and contact modelling}

\subsubsection{Geometry of the problem and FE model}

The structural problem investigated in this study is shown in Fig. 1.

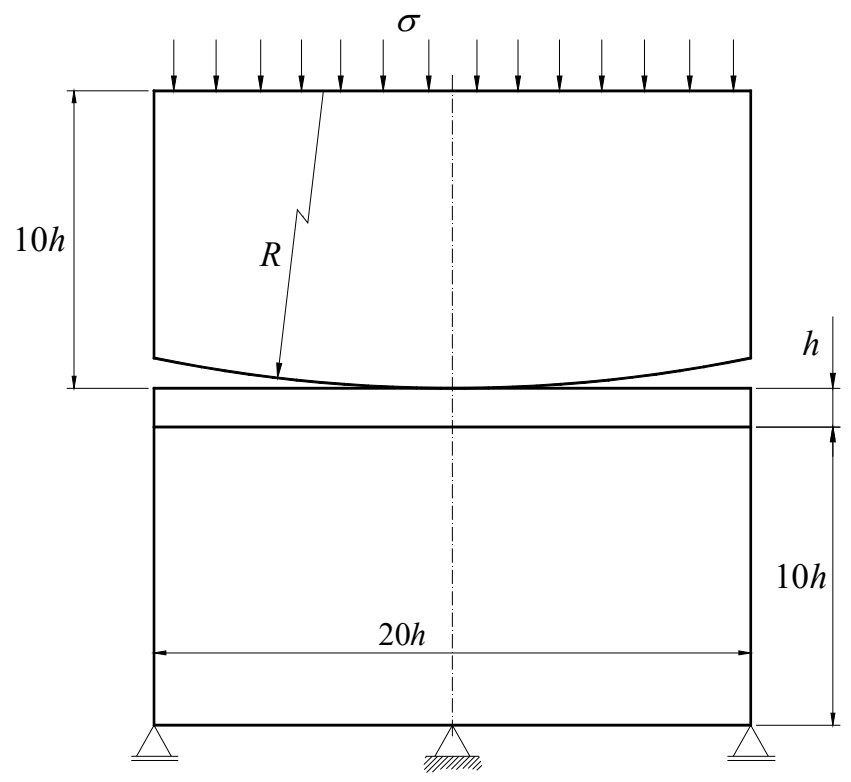

Fig. 1 Geometry of the FEM model

The material of all three contacting bodies is defined as isotropic and linearly elastic and the model is solved by using the nonlinear static analysis in the Femap software package. The model is meshed with plane strain finite elements and load is defined as a uniformly distributed stress $\sigma=10^{8} \mathrm{~N} / \mathrm{mm}$ acting in the negative $y$ direction on the top surface of the indenter. 
The behaviour of the structure shown in Figure 1 is studied from three aspects: (i) the influence of geometry (by varying the indenter radius), (ii) the influence of material properties (by changing elastic constants of each body in turn) and (iii) the influence of load intensity. Two principal contact parameters, peak contact pressures and contact widths, are determined for each of the above mentioned variations of the model. In doing that, geometry variation is the reference frame within which all other analyses are carried out and their results compared.

As already stated above, different geometries are modelled only by the variation of the indenter radius $R$, while the remaining measures are kept in the same proportion as shown in Fig. 1. Geometries for ratios $R / h=50,100,200$ and 500 have been investigated. A referential analysis of each geometry is taken for the case when all three bodies have the same elastic properties: $E=200 \mathrm{GPa}, v=0.3$. A detail of the mesh in the vicinity of the initial point of contact is shown in Fig. 2, clearly showing the uniform structuring of the mesh in the region where accuracy of the result is of utmost importance.

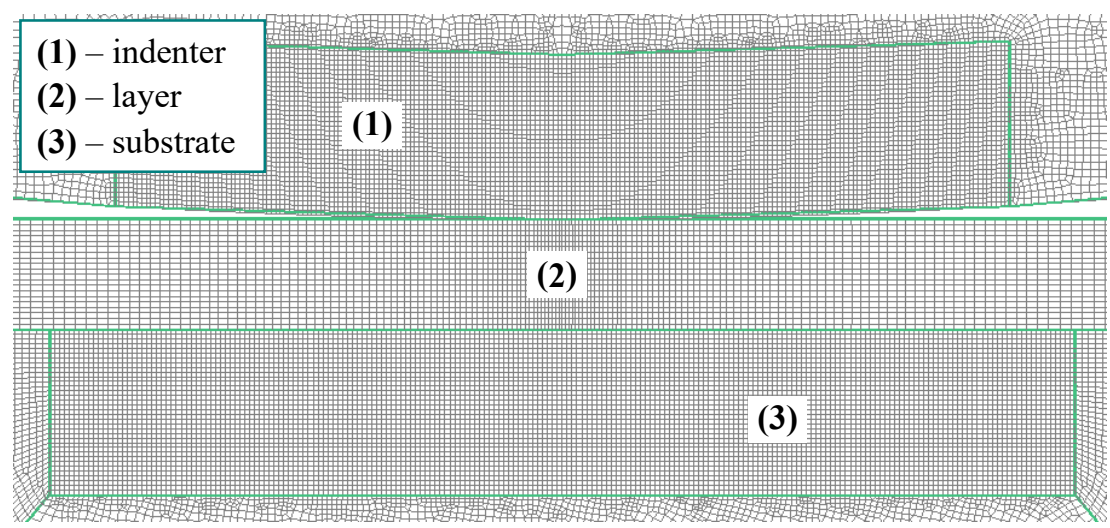

Fig. 2 Mesh detail around the initial point of contact

\subsubsection{Contact modelling in Femap}

In order to analyse the transmission of forces between the contacting bodies shown in Fig. 2, a slide line element was used as a much more versatile and flexible element property available in Femap compared to the limiting capabilities of gap elements. Slide line elements have the ability to deal with non-conforming meshing (node-on-element) and also allow large relative motion of the contacting surfaces in the tangential direction (i.e. along the slide line). Every slide line element can contain an arbitrary number of nodes lying on the lines/curves of both bodies that are in contact or that are expected to get into contact. The nodes of one body are designated as the master nodes and the nodes of the other as the slave nodes. A three-node (two master nodes $\mathrm{M}_{1}, \mathrm{M}_{2}$ and one slave node $\mathrm{S}$ ) slide line element is shown in Fig. 3.



Fig. 3 Three-node slide line element: $\xi, \xi_{0}$ - current and previous surface coordinate, $\delta_{\mathrm{n}}$ - penetration of slave node into the master segment, $\delta_{\mathrm{t}}$ - sliding of the slave node on the master segment 
The line segments joining two neighbouring slave or master nodes are called the slave or master segments. By default, the contact algorithm checks slave nodes for penetration into the master segments (see Fig. 3), which is referred to as unsymmetrical penetration. If better accuracy of the result is desired, both slave and master nodes can be checked for penetration, which is then called symmetric penetration [24-25]. The algorithm uses the penalty method in imposing the displacement compatibility condition and calculating the contact forces.

\subsection{Finite element analysis results}

Distributions of contact pressures $p_{\mathrm{n} 1}$ at the indenter-layer interface and $p_{\mathrm{n} 2}$ at the layersubstrate interface are obtained as nodal values of the $\sigma_{\mathrm{y}}$ stresses from the FEM model, and for the initial geometry of $R / h=50$ they are shown in Fig. 4 in dimensionless form. Hereafter, quantity $a$ will denote the half-width of the indenter-layer contact and quantity $b$ will denote the half-width of the layer-substrate contact.


Fig. 4 Contact pressure distributions for the initial geometry $R / h=50$ : a) at the indenter-layer interface $\left.p_{\mathrm{n} 1, \max } / \sigma \approx 11 ; a / h \approx 1.29 ; \mathrm{b}\right)$ at the layer-substrate interface $p_{\mathrm{n} 2, \max } / \sigma \approx 9.35 ; b / h \approx 1.78$

As can be seen from Fig. 4, the forces between the indenter and the layer are transmitted over a significantly smaller area with a markedly higher maximal contact pressure. It is an expected result, which is in agreement with results from the available literature. Qualitatively similar results are obtained for the remaining $R / h$ geometries (see diagrams in Appendix).

\subsubsection{Influence of load intensity and indenter radius}

If external load is applied gradually, the contact areas will gradually increase along with the peak contact pressures. This is to be expected since the geometry of the indenter forms a non-conforming contact with the upper surface of the layer. This phenomenon has not been investigated adequately in existing literature for the case of elasticity of all contacting bodies.


Fig. 5 Contact stresses for different load intensities: a) indenter-layer interface, b) layer-substrate interface 
Each geometry $R / h$ was investigated for a series of gradually increasing loads $\sigma_{\mathrm{gr}}$, namely $0.2 \sigma ; 0.4 \sigma ; 0.6 \sigma ; 0.8 \sigma$ and $\sigma$. Fig. 5 shows contact pressure distributions for these loads for geometry $R / h=50$. Qualitatively similar diagrams to those presented in Fig. 5 are obtained for the remaining geometries (see diagrams in Appendix) and the consolidation of the results for maximal contact pressures and contact half-widths for all $R / h$ geometries with respect to load intensity produces the diagrams shown in Figs 6 and 7.
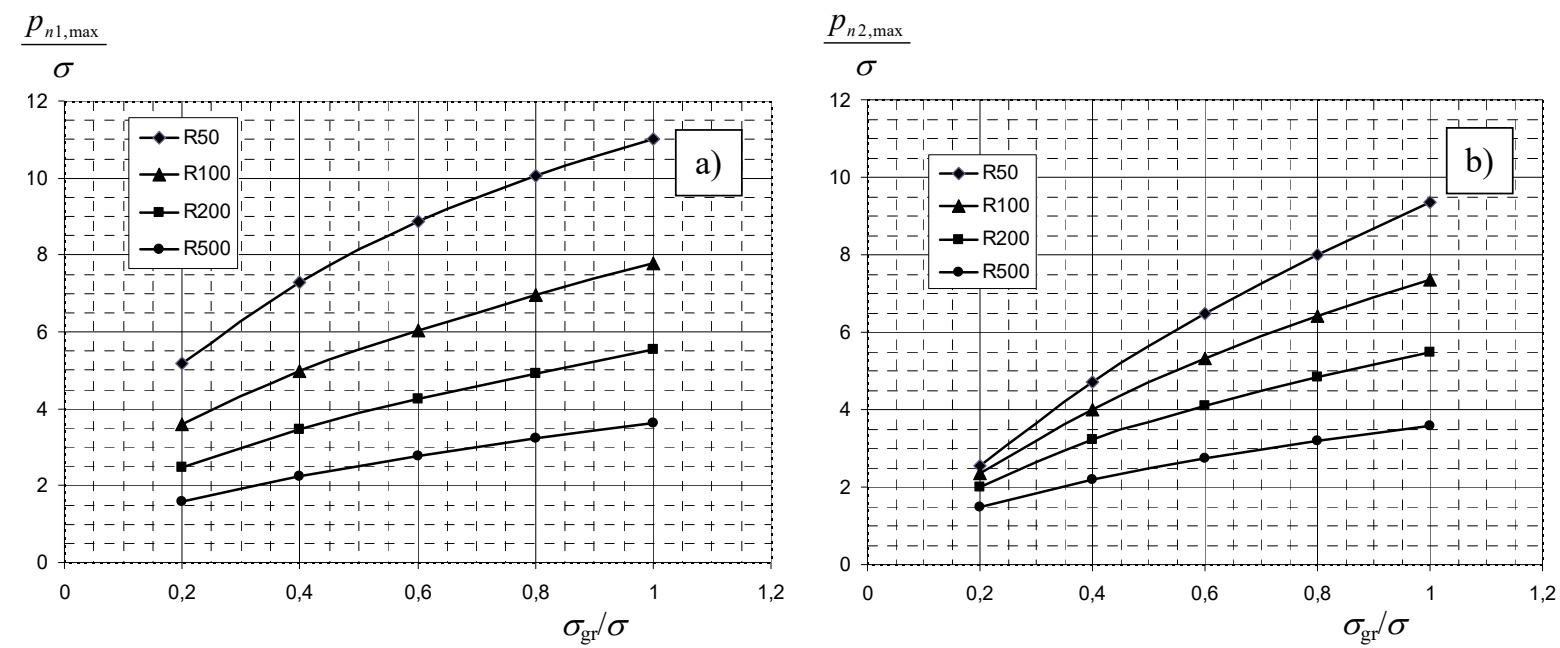

Fig. 6 Variation of maximal contact pressures with load intensity for all $R / h$ geometries; a) at the indenter-layer interface, b) at the layer-substrate interface
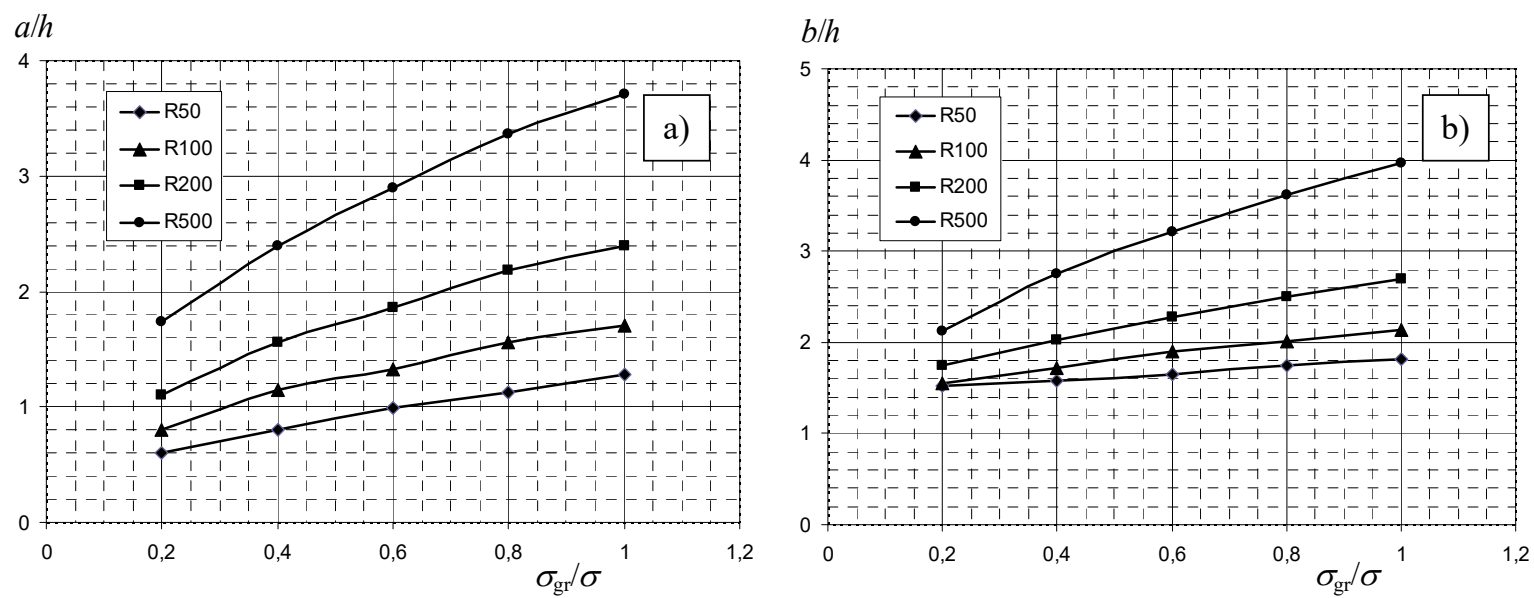

Fig. 7 Variation of contact half-width with load intensity for all $R / h$ geometries; a) at the indenter-layer interface, b) at the layer-substrate interface

Figs 6 and 7 clearly show that maximal contact pressures and contact half-widths nonlinearly depend on the intensity of the applied load. This nonlinearity is not, however, very pronounced and for very high ratios $R / h$ these functions approach linearity for maximal contact pressures, while for smaller $R / h$ the functions of contact half-widths become almost perfectly linear. Fig. 7 indicates that the change in size of the contact area is actually very significant as the load increases, which to the best of the authors' knowledge is an aspect of receding contacts in layered structures not previously investigated in literature. Furthermore, it is also evident from Fig. 7 that the effect of load on the contact area gradually vanishes with the values of $R / h$ decreasing (observe the curve slopes in Fig. 7).

There is an inherent duality in the presented results in that in the cases of both higher and lower values of the ratio $R / h$ they simultaneously contradict and conform to the classical assumptions and simplifications about receding contacts in layered structures. Concentrated loads, flat punches and pressure distributions of constant width had been used extensively by 
numerous authors in the past in order to model the indentation of the layer in receding contacts - for that reason the conventional approach was to regard the extent of the contact area as independent of the level of loading, while the contact stresses were understood to linearly depend on load intensity $[1,6,7,18]$. In contrast to that, by increasing $R / h$ in the case of a cylindrical indenter the model asymptotically approaches the case of a flat punch as the limit case (i.e. $R=\infty$ ) of a cylindrical indenter and, while nearly obeying the assumption of linear dependence of contact pressures, the results at the same time markedly diverge from classical assumptions by rapid expansion of the contact area as external load increases. On the other hand, by decreasing $R / h$ the model asymptotically approaches the case of a concentrated force (or a narrowly distributed pressure) on top of the layer - in this case, although the invariability of the contact area is increasingly evident with smaller $R / h$, the results diverge again from the classical assumptions by nonlinear dependence between contact pressures and load.

Finally, the influence of the indenter radius on contact parameters is displayed in Fig. 8 for the case of full load intensity, showing how the values gradually converge.
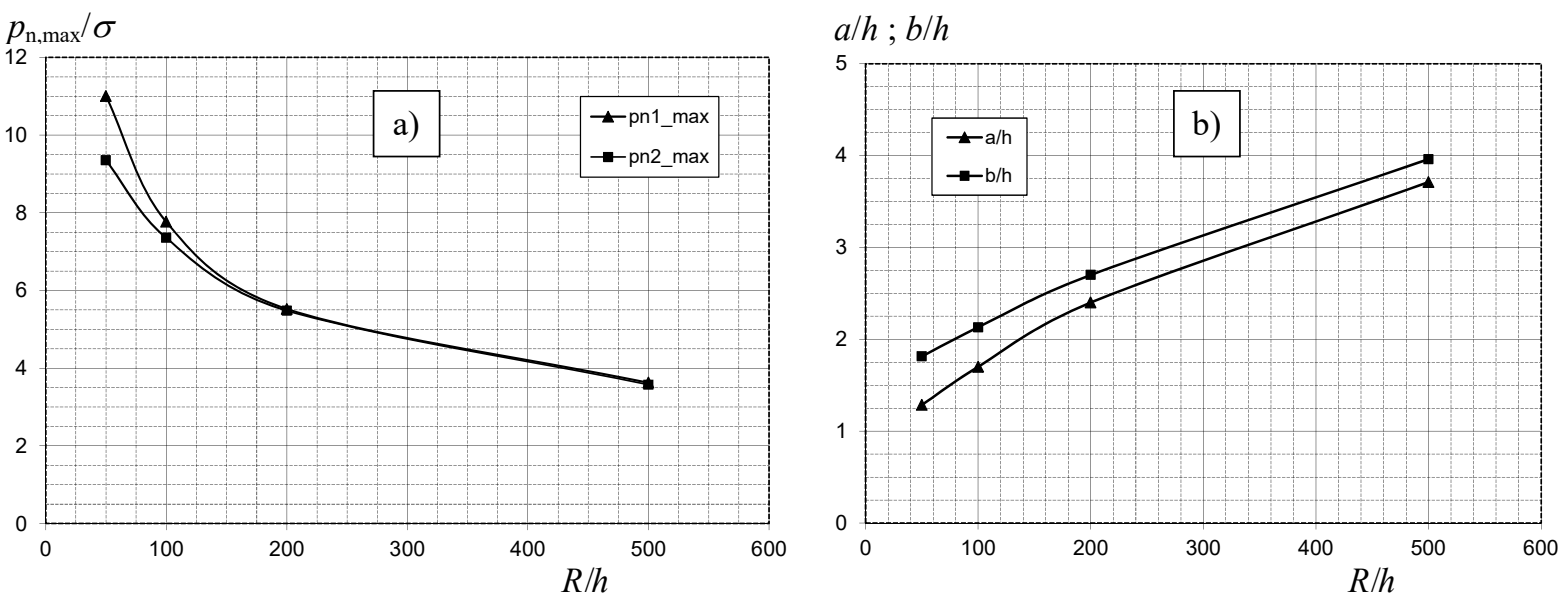

Fig. 8 Values of (a) maximal contact pressures and (b) contact half-widths with respect to $R / h$ at full load

The curves in Fig. 8 are obtained from the values of the rightmost points (full load level) shown in Figs 6 and 7, with respect to $R / h$. Considering the geometry and physics of the problem, as well as the results presented in Figs 6 and 7, this is an expected result. The contact pressures converge more rapidly than the half-widths $a$ and $b$ and for $R / h=500$ the difference between the two contact pressures is approximately 1.45\%, while half-widths $a$ and $b$ differ by approximately $6.36 \%$.

\subsubsection{Influence of material properties}

Keeping in mind that the preceding considerations were restricted to the referential analyses of material similarity, it is also of interest to investigate the effect of material properties. For this purpose, the material properties of each of the three bodies were varied individually as shown in Table 1, while the properties of the remaining two bodies were kept at the referential values $(E=200 \mathrm{GPa}, v=0.3)$.

Table 1 Variations of material properties

\begin{tabular}{|c|c|c|c|}
\hline Quantity & Analysis 1 & Analysis 2 & Analysis 3 \\
\hline $\begin{array}{c}\text { Modulus of elasticity, } \\
\text { E/ GPa }\end{array}$ & 110 & 70 & 3 \\
\hline Poisson's number, $v$ & 0.32 & 0.335 & 0.36 \\
\hline
\end{tabular}


The values in Table 1 are taken in this particular combination so as to correspond to the values used by Iyer in his FEM analysis given in [26] for titanium, aluminium and epoxy resin, which are widely encountered in pin-plate connections in aviation industry. Interference/zeroclearance pin-plate connections are another class of receding contacts which are also studied by the authors in a forthcoming publication.

Three materials for each of the three bodies yield nine simulations for each of the four investigated geometries. This finally gives a total of 36 possible models and simulations, but it must be noted that not all of them were carried out. In several cases the convergence of the model was not possible due to the excessive compliance of the model in the case of the very small value of $E$. Therefore, some diagrams lack the curves pertaining to analysis 3 (as designated in Table 1) since the FEM model failed to produce good results. Figs 9-11 present the resulting pressure distributions for geometry $R / h=50$, and qualitatively similar results for the remaining geometries are shown in Appendix.
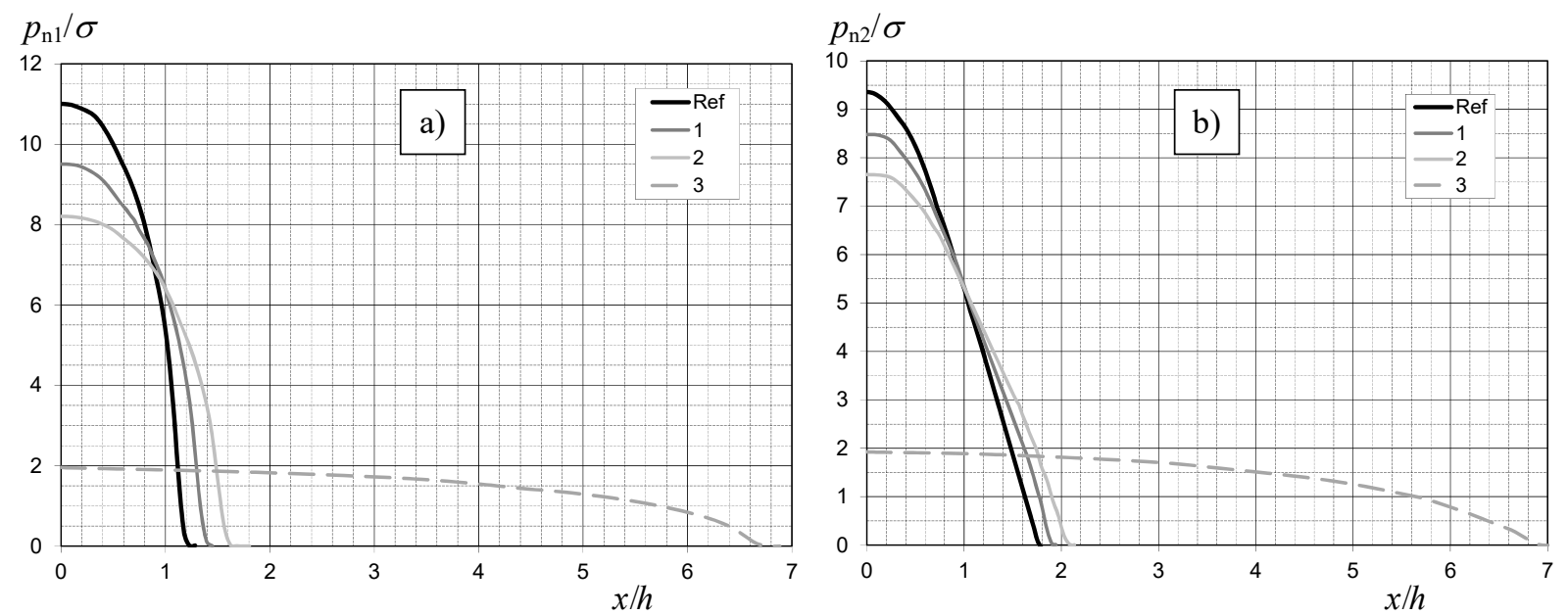

Fig. 9 Contact pressure distributions for different material properties of the indenter; a) at the indenter-layer interface, b) at the layer-substrate interface


Fig. 10 Contact pressure distributions for different material properties of the layer;

a) at the indenter-layer interface, b) at the layer-substrate interface 

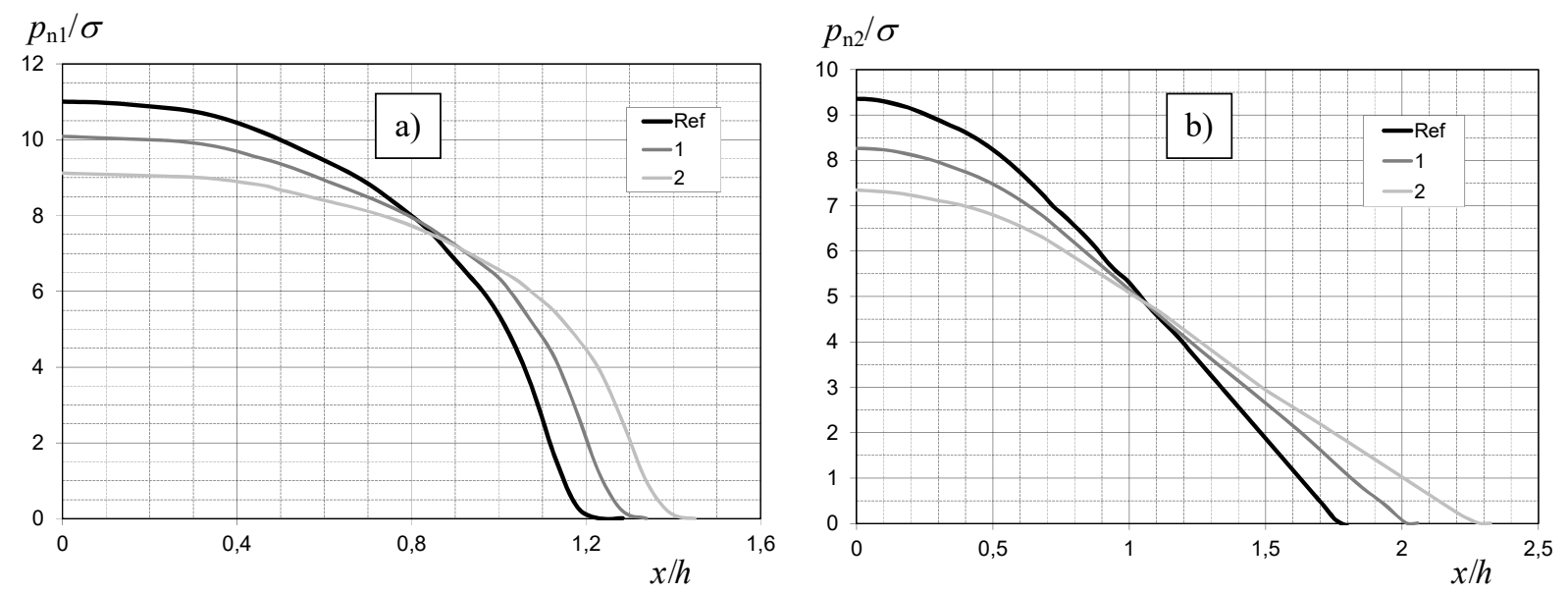

Fig. 11 Contact pressure distributions for different material properties of the substrate; a) at the indenter-layer interface, b) at the layer-substrate interface

The following conclusions can be drawn from Figs 9-11:

A. Lower values of modulus of elasticity of any of the three bodies produce larger contact areas and lower maximal values of contact pressures in comparison to the case of material similarity in the referential model.

B. The indenter material exerts the greatest influence on the contact parameters at the indenter-layer interface.

C. The substrate material exerts the greatest influence on the contact parameters at the layer-substrate interface.

D. The layer exerts the weakest influence on the contact par. on either contact surface.

\subsection{Comparison with existing results}

A comparison of the obtained results with the results published in the available literature can be carried out only to the extent of similarity between the already existing research and the results obtained in this paper. As already emphasized, the presented results are mainly oriented toward uninvestigated aspects of behaviour of receding contacts in layered structures, so this comparison is somewhat approximate and limited in scope. This is all the more true since the usual approach is to simplify the model by introducing concentrated loads, by assuming constant load width or by modelling the indenter and/or support as rigid. These simplifications lend themselves to the variety of analytical approaches often used in literature, however, the numerical studies have not been as comprehensive as might be expected either.

As a suitable reference for comparison, from the geometry aspect of the problem and the aspect of material similarity, we shall use the results presented by Comez in [19] (figures 8 and 9 in the paper) for the case of $R / h=500$. In order to compare the contact parameters in this particular case we must first recalculate the dimensionless quantities $p(x) /(P / h)$ and $G /(P / h)$ from [19] (with $P$ being the force and $G$ shear modulus) in order to correlate them with the quantities used here. Taking the material properties used in the referential analysis in this paper into account it is easy to calculate that the results in [19] must be compared to the results for $R / h=500$ at load level $\sigma_{\mathrm{gr}}=0.385$. Contact parameters for this load level are obtained from diagrams in Figs 6 and 7 and they are summarized with the results from [19] in Table 2.

Table 2 Comparison with the results of Comez in [19]

\begin{tabular}{|c|c|c|c|c|}
\hline Quantity & $a / h$ & $p_{\mathrm{n} 1, \max } /(P / h)$ & $b / h$ & $p_{\mathrm{n} 2, \max } /(P / h)$ \\
\hline Present paper & $\mathbf{2 . 3 5}$ & $\mathbf{0 . 2 3}$ & $\mathbf{2 . 7}$ & $\mathbf{0 . 2 1 5}$ \\
\hline Comez [19] & 1.74 & 0.35 & 2.19 & 0.34 \\
\hline
\end{tabular}


The values given in Table 2, although approximate, clearly show noticeable differences between the results. The principal difference in the approach in the present analysis is the elasticity of the indenter, whose deformation greatly contributes to the widening of both contact areas and consequently to a decrease in contact pressures. If the indenter is assumed rigid, upon loading all points would be displaced by the same amount, thus preserving the undeformed shape of the body. With elasticity taken into account the absolute value of the vertical displacement for any given load is the lowest at the indenter's tip point (i.e. the middle point of the circular edge); for instance, at the load level of $0.4 \sigma$ the outermost point of the circular edge undertakes a vertical displacement about 2.09 times larger than the displacement of the middle point, and this difference steadily progresses as the load increases.

\section{Experimental analysis}

\subsection{Experimental model and measuring equipment}

The experiment was carried out on a steel model manufactured for the specific purpose of this experiment, and the components of this model are shown in Fig. 12.

Loading of the model was carried out on a hydraulic powered static testing machine Messphysik Beta 50-5. The two lead screws of the testing machine are about 420 $\mathrm{mm}$ apart, which was the rough estimate for the upper width limit of the model.

The measurement method used in this experiment relies on the principle of digital image correlation (DIC) and for that purpose the optical system ARAMIS $4 \mathrm{M}$ was used, with the ability to measure both displacements and strains. This DIC system employs a 16-bit CMOS (Complementary Metal-Oxide-Semiconductor) camera with a one inch sensor.

The most important measurement characteristics of this system are given in Table 3 .

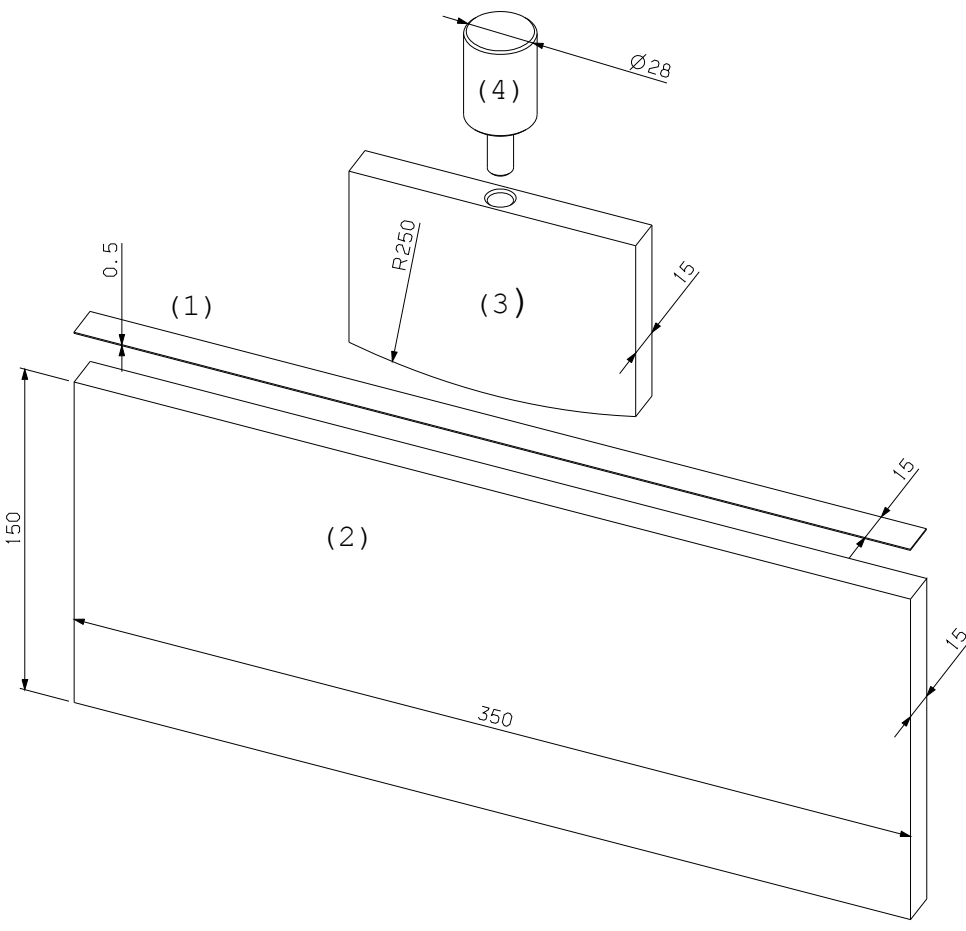

Fig. 12 Experimental model: (1) layer, (2) substrate, (3) cylindrical indenter, (4) shoulder for the grip of the testing machine

Table 3 Main measurement characteristics of the ARAMIS 4M system [27]

\begin{tabular}{|c|c|c|c|c|c|}
\hline $\begin{array}{c}\text { Measuring } \\
\text { volume, }[\mathrm{mm}]\end{array}$ & $\begin{array}{c}\text { Camera resolution, } \\
{[\text { pixel] }}\end{array}$ & $\begin{array}{c}\text { Maximal frame } \\
\text { rate, }[\mathrm{Hz}]\end{array}$ & Shutter time & $\begin{array}{c}\text { Strain } \\
\text { measuring range }\end{array}$ & Strain accuracy \\
\hline $\begin{array}{c}10 \times 7 \ldots \\
4000 \times 2700\end{array}$ & $2352 \times 1728$ & 60 & $0.1 \mathrm{~ms} \ldots 2 \mathrm{~s}$ & $\begin{array}{c}0.02 \% \ldots \\
>100 \%\end{array}$ & up to $0.02 \%$ \\
\hline
\end{tabular}

Deformation and motion on the surface of the specimen can be detected provided that the surface is prepared by applying a stochastic speckle pattern (sprayed black and white colour) on the surface. Digital image correlation relies in essence on the ability of the software to compare sequences of digital images and deduce the displacements based on the calculated differences between the images in the sequence. 


\subsection{Experimental procedure and results}

\subsubsection{Preparation of the experiment}

The model was prepared first by spraying a stochastic pattern (or raster) on the side surfaces of all three bodies in the area surrounding the initial point of contact of the indenter. Since the side surface of the layer is only $0.5 \mathrm{~mm}$ thick, the measuring volume (area on which the measurement is done) had to be appropriately small.

The first step in the creation of the pattern is the application of white paint (background colour), on top of which black colour is sprayed, thus forming a stochastic pattern. Since the measuring volume must be small enough to enable the tracking of layer deformation, a speckle pattern of appropriate uniformity and density is required - i.e. the sprayed dots of the black paint must be small enough and must be dispersed in a reasonably uniform manner. For this task the black toner MicroGraphics ${ }^{\mathrm{TM}}$ for laser printers was used. The procedure is as follows: (1) the black toner is mixed with alcohol; (2) the mixture is homogenized for about $40 \mathrm{~s}$ by using ultrasound in the device Ultrasonic cleaner; (3) the thus obtained homogenized liquid is poured into a pneumatic Iwata High Performance Microbrush, which uses compressed air from a compressor tank to finely disperse the black colour on the white background colour.

The next step consists of adjusting the distance of the camera and focus of the lens. The measuring system is then calibrated for the measuring volume of $12 \times 8 \mathrm{~mm}$ using the standard manufacturer's calibration object (calibration cube) CQ10 $\times 8$.

In the final step three reference measurement points are defined on the layer within the ARAMIS software, as shown in Fig. 13. The displacements of these measurement points uniquely characterize the deformation behaviour of the layer.



Fig. 13 Positions of characteristic points on the layer

Software and hardware settings used for this experiment are the following: measuring volume $12 \times 8 \mathrm{~mm}$, frame rate 1 frame/s, facet size $30 \times 30$ pixels, facet step $20 \times 20$ pixels, image resolution $2352 \times 1728$ pixels, 16 bit colour resolution.

\subsection{Measurement and comparison with numerical results}

The deformation behaviour of the layer was tracked while the compressive force was uniformly increased on the testing machine from 0 to $1500 \mathrm{~N}$ during a period of 35 seconds, followed by the phase of unloading. The positions of the three measurement points were recorded for each frame (intervals of $1 \mathrm{~s}$ ) and the obtained displacement-time curves are shown in Fig. 14. The calculation of load intensity was straightforward for each frame (load stage) since the testing machine and the ARAMIS software were synchronized during operation.

The maximal load intensity of $1500 \mathrm{~N}$ was determined as an approximate value for which yielding in the layer material might be expected. Although this is a non-Hertzian contact problem, this value was estimated by using the Hertz theory as the first approximation for the simple purpose of framing the parameters of the experiment.

The results of the DIC measurement are shown in Fig. 14. 


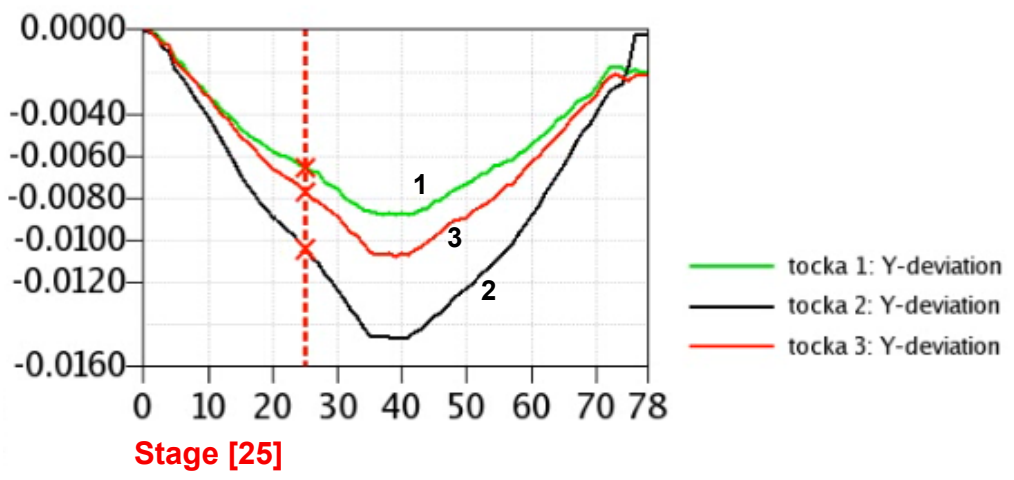

Fig. 14 Measured vertical displacements ( $y$ direction) of three reference points; e.g. at stage 25 (after $25 \mathrm{~s}$ ) the force equals approximately $1070 \mathrm{~N}$

Load-displacement curves given in Fig. 14 show that the vertical displacement at point 2 is greater than the displacements measured at points 1 and 3. This is in good agreement with the behaviour of unbonded layers, in which external load ensues separation between the layer and the substrate and to the best of the authors' knowledge on this subject these results represent the first known DIC-based measurement of layer behaviour in receding contacts.

The measured displacements of reference points 1 and 3 are not identical, which must be attributed to the initial imperfections in the layer geometry and the inability to define these two points in a perfectly symmetrical configuration, since the points have to be selected manually. Therefore, for the purpose of comparison with the results obtained by the FEM analysis an approximate mean value between displacements 1 and 3 will be used.

A FEM model of the presented experimental model was constructed in Femap, and the displacements in the $y$ direction for the nodes corresponding to the reference points 1 and 3 were compared. Displacements from the numerical model were taken for the load levels that correspond to the resultant force level of 500, 1000 and $1500 \mathrm{~N}$. As a measure of comparison the difference of displacements $\Delta y=y_{2 / 3}-y_{1}$ is taken and summarized in Table 4.

Table 4 Comparison for $\Delta y=y_{2 / 3}-y_{1}$ between the experiment and FEM

\begin{tabular}{|c|c|c|c|}
\hline Load level & $500 \mathrm{~N}$ & $1000 \mathrm{~N}$ & $1500 \mathrm{~N}$ \\
\hline Experiment, $\Delta y_{\mathrm{E}}$ & 1.4 & 2.6 & 5 \\
\hline FEM, $\Delta y_{\mathrm{F}}$ & 1.38 & 2.44 & 3.5 \\
\hline$\left(1-\Delta y_{\mathrm{F}} / \Delta y_{\mathrm{E}}\right) \cdot 100 \%$ & $1.43 \%$ & $6.15 \%$ & $30 \%$ \\
\hline
\end{tabular}

For lower values of the force the results obtained from the FEM model are in very good agreement with the experimental data. As the load increases, experimentally measured displacements tend to diverge from the FEM results. This can be attributed to two aspects of the experiment. Firstly, for the layer it was not possible to use a steel sheet that would be perfectly straight, which could significantly influence the behaviour and the final deformed shape of the layer. Secondly, considering the layer was quite thin $(0.5 \mathrm{~mm})$, it is likely that plastic deformations took place in the layer, given the fact that contact stresses dominate the stress field and that the stresses within the body can exceed the yielding point at seemingly modest load levels.

\section{Conclusion and outlook}

The behaviour of receding contacts in structures with a single unbonded layer was studied from three aspects: (i) the influence of geometry (i.e. ratio $R / h$ ), (ii) the influence of load level and (iii) the influence of material properties. The problem was considered within the frame of linear elasticity, all three bodies (indenter, layer and substrate) were considered 
as elastic and plane strain was assumed for all numerical models. The obtained results show that the elastic indenter greatly influences the contact parameters and the obtained results markedly diverge from the solutions and models where the indenter is modelled as rigid, or where the indenter is replaced by a concentrated load or a predefined load distribution. Maximal contact pressures and contact half-widths prove in general to be nonlinear functions of load intensity, but the effect of this nonlinearity is not very pronounced and tends to disappear for large $R / h$ when it comes to maximal contact pressures, as well as for small $R / h$ when it comes to contact half-widths. These results present a novelty in this field of study and should be scrutinized in future research. When material dissimilarity is considered, it has been found that lower values of elastic constants of any of the three bodies in the model lead to the widening of contact areas and to a significant drop in maximal contact stresses. Furthermore, it has been shown that the indenter material exerts the greatest influence on the contact parameters at the indenter-layer interface, while the substrate material exerts the greatest influence on the contact parameters at the layer-substrate interface.

The experimental measurements support the validity of numerical models and the obtained results. The experimental data and the results obtained by FEM are in very good agreement as long as elastic stresses can be assumed with certainty. For loads that approximately correspond to the emergence of yielding in the material, the results diverge significantly.

Future research should be steered toward a more detailed investigation into material dissimilarity in the context of the results already obtained for the case of material similarity. In doing so, it would be possible to characterize the behaviour of receding contacts in layered structures in the entirety of its influencing factors, which lends itself to the formulation of approximate mathematical expressions that could be used as universally valid for such structures. In addition, the case involving friction on the contacting surfaces could also be considered as a further improvement to the model. Finally, since the described experiment was the very first experiment of this sort to be carried out, a repeated experiment with different, and perhaps bulkier layers could be undertaken. This does impose certain financial and logistic limitations as to the massiveness of the structure that would be tested, but it might provide additional insight into the validity and scope of application of the presented results.

\section{APPENDIX}

Appendix A Contact pressure distributions for different load levels
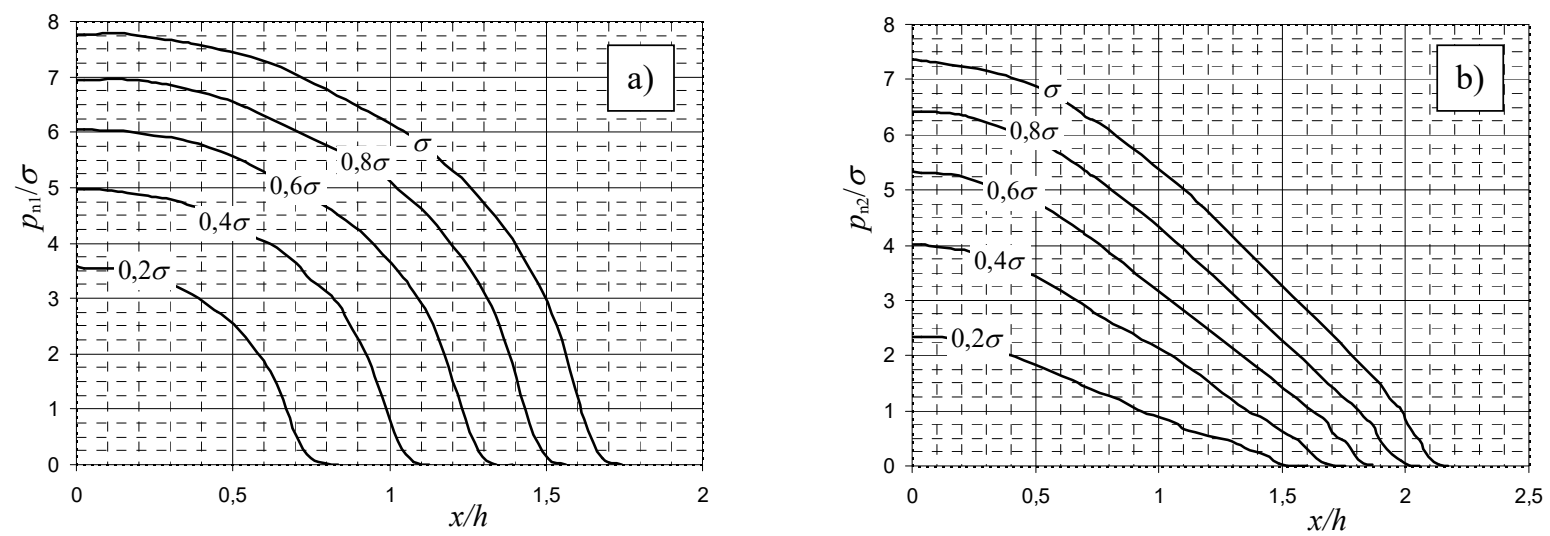

Fig. A1 Contact stresses for different load intensities for $R / h=100$ : a) indenter-layer interface, b) layer-substrate interface 

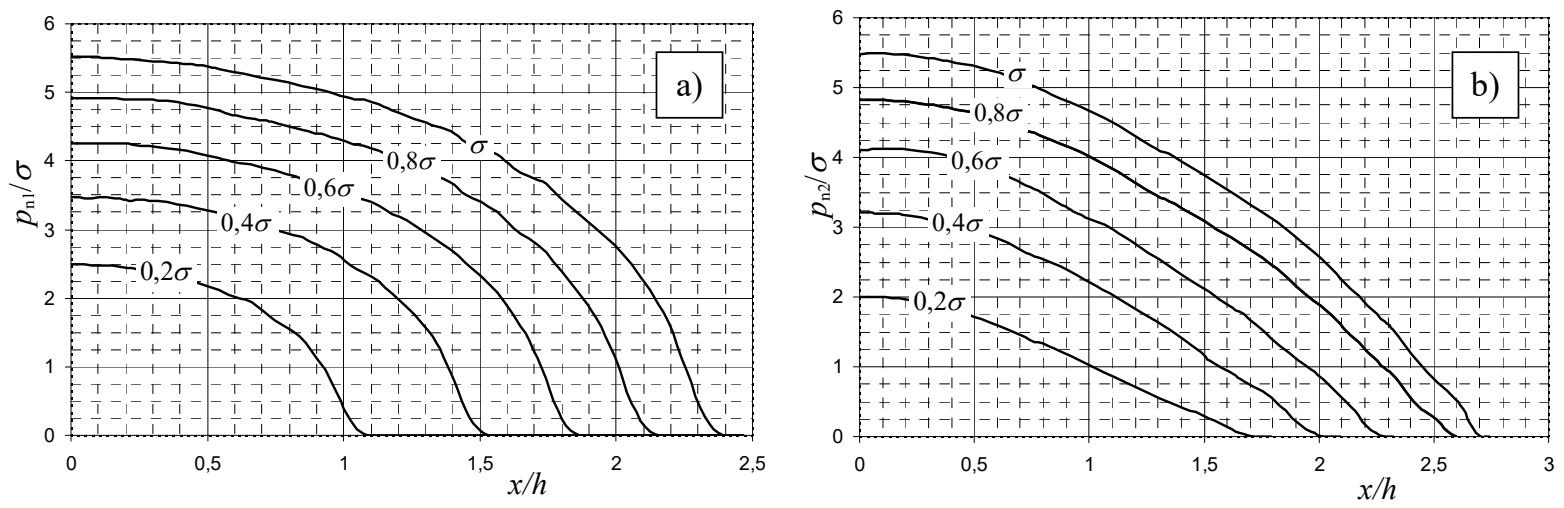

Fig. A2 Contact stresses for different load intensities for $R / h=200$ : a) indenter-layer interface, b) layer-substrate interface


Fig. A3 Contact stresses for different load intensities for $R / h=500$ : a) indenter-layer interface, b) layer-substrate interface

Appendix B Contact pressure distributions for varying material properties
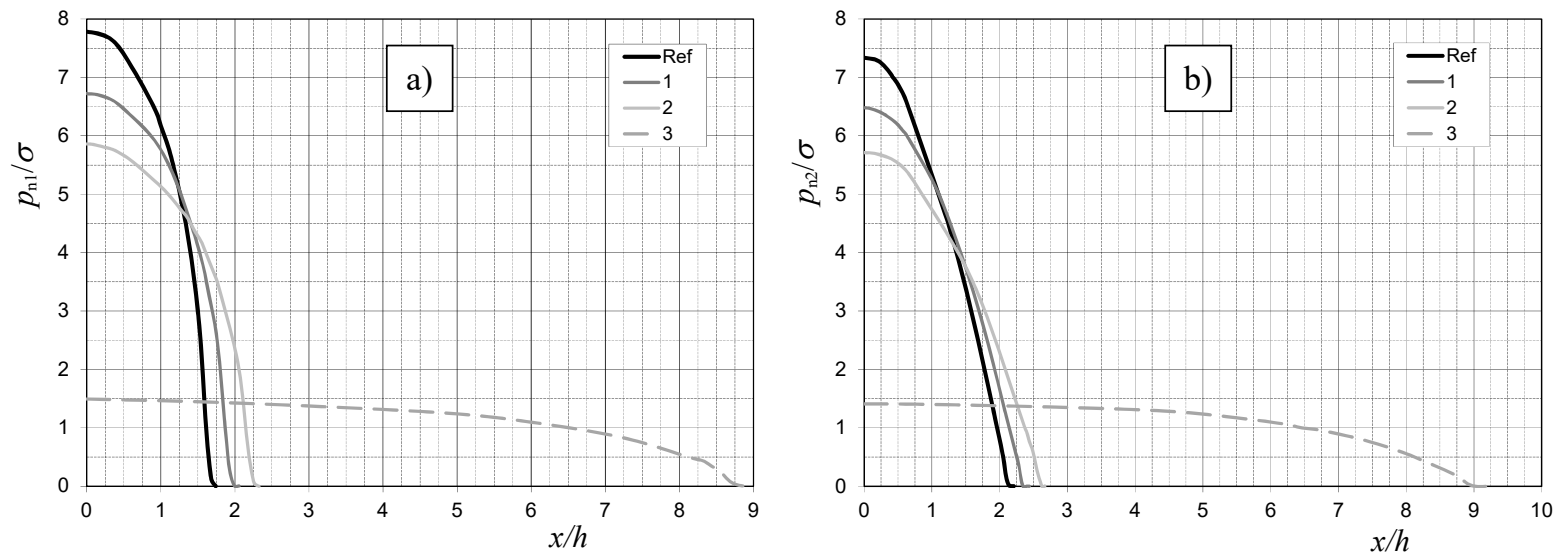

Fig. B1 Contact pressure distributions for different material properties of the indenter for $R / h=100$; a) at the indenter-layer interface, b) at the layer-substrate interface 

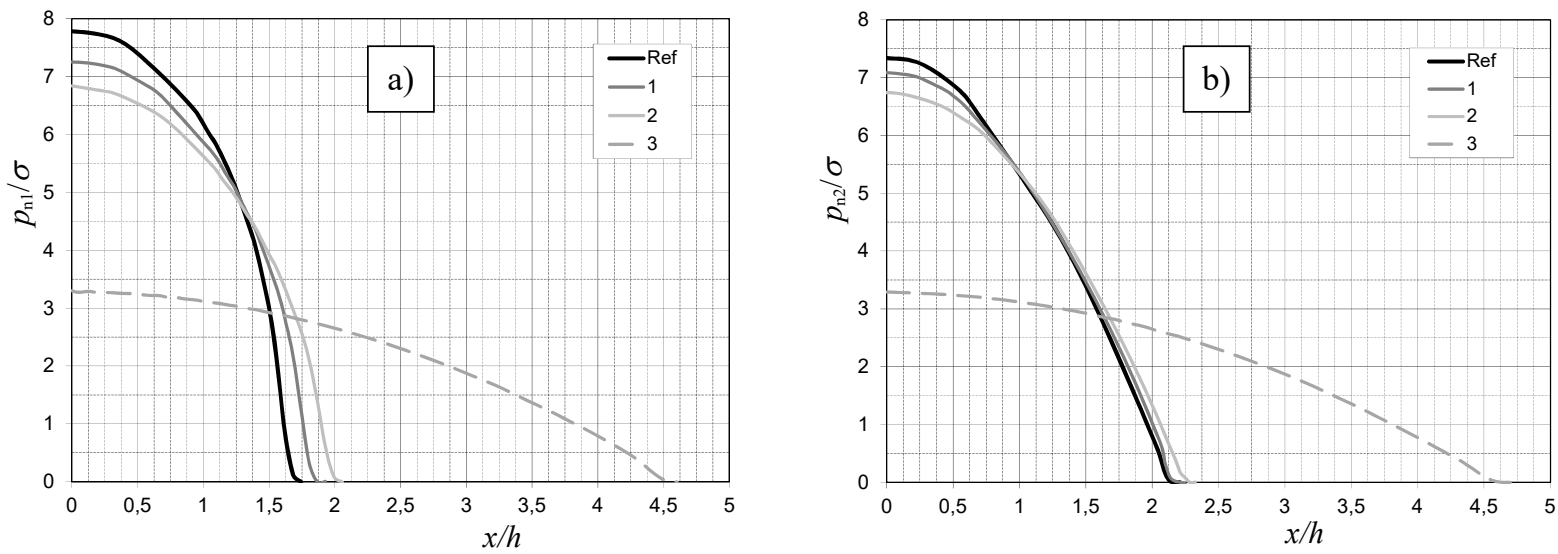

Fig. B2 Contact pressure distributions for different material properties of the layer for $R / h=100$; a) at the indenter-layer interface, b) at the layer-substrate interface
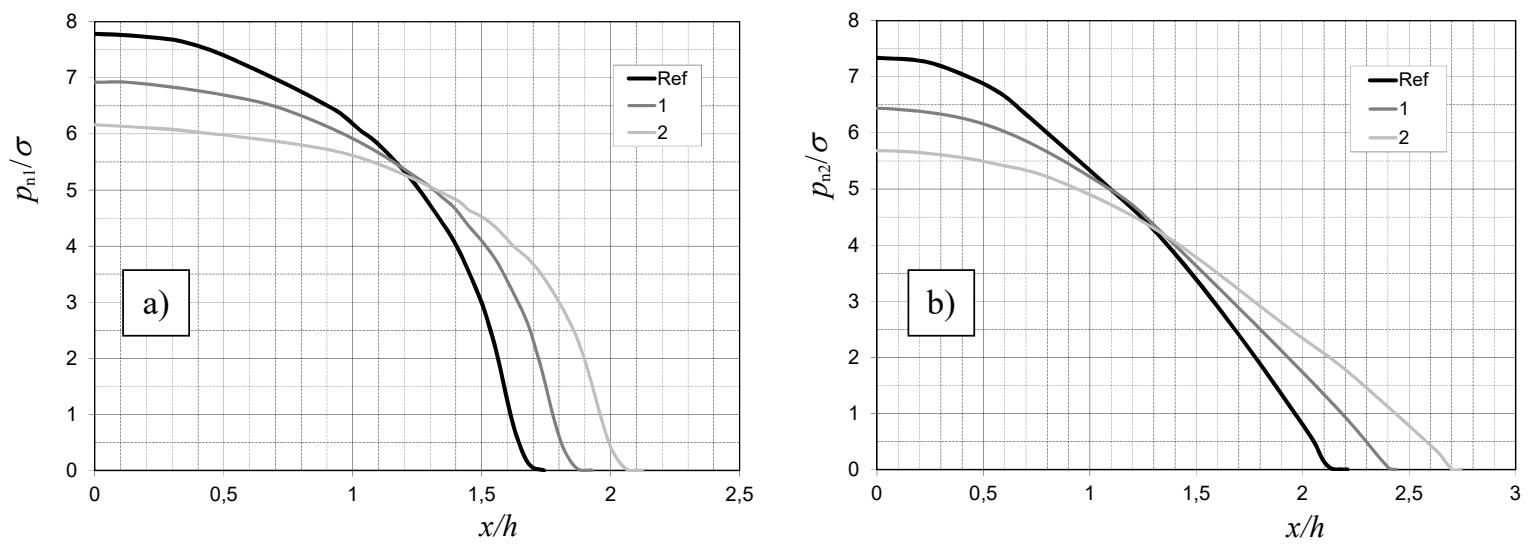

Fig. B3 Contact pressure distributions for different material properties of the substrate for $R / h=100$; a) at the indenter-layer interface, b) at the layer-substrate interface


Fig. B4 Contact pressure distributions for different material properties of the indenter for $R / h=200$; a) at the indenter-layer interface, b) at the layer-substrate interface 

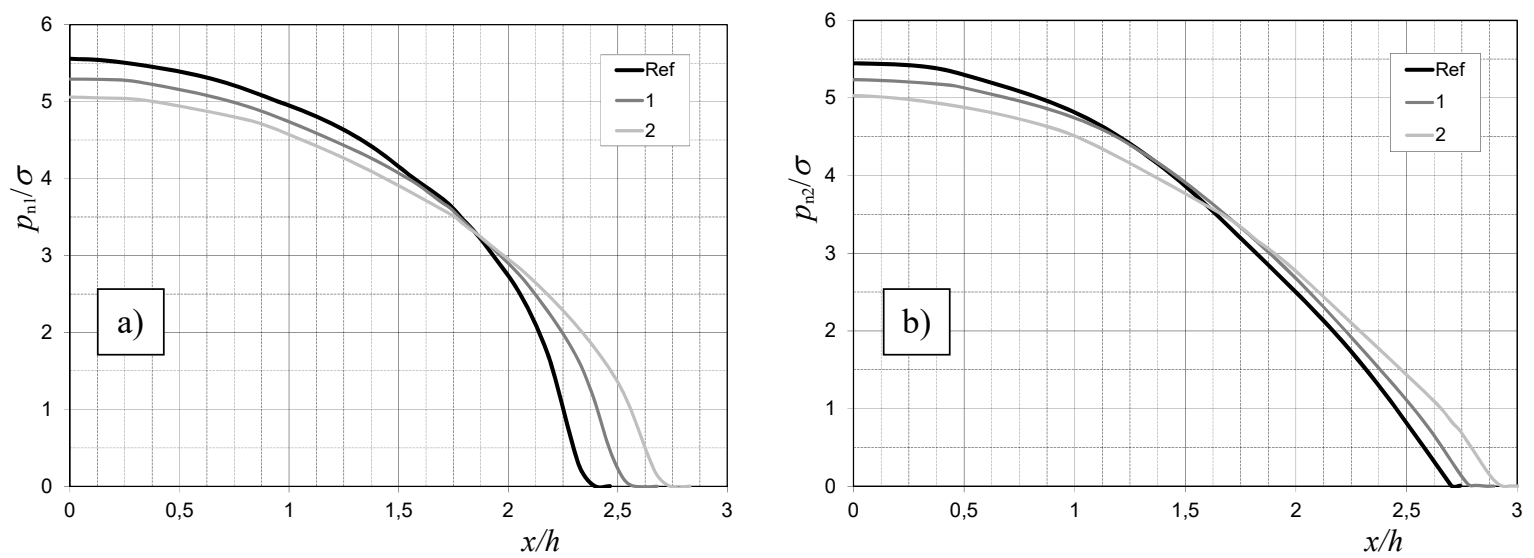

Fig. B5 Contact pressure distributions for different material properties of the layer for $R / h=200$; a) at the indenter-layer interface, b) at the layer-substrate interface
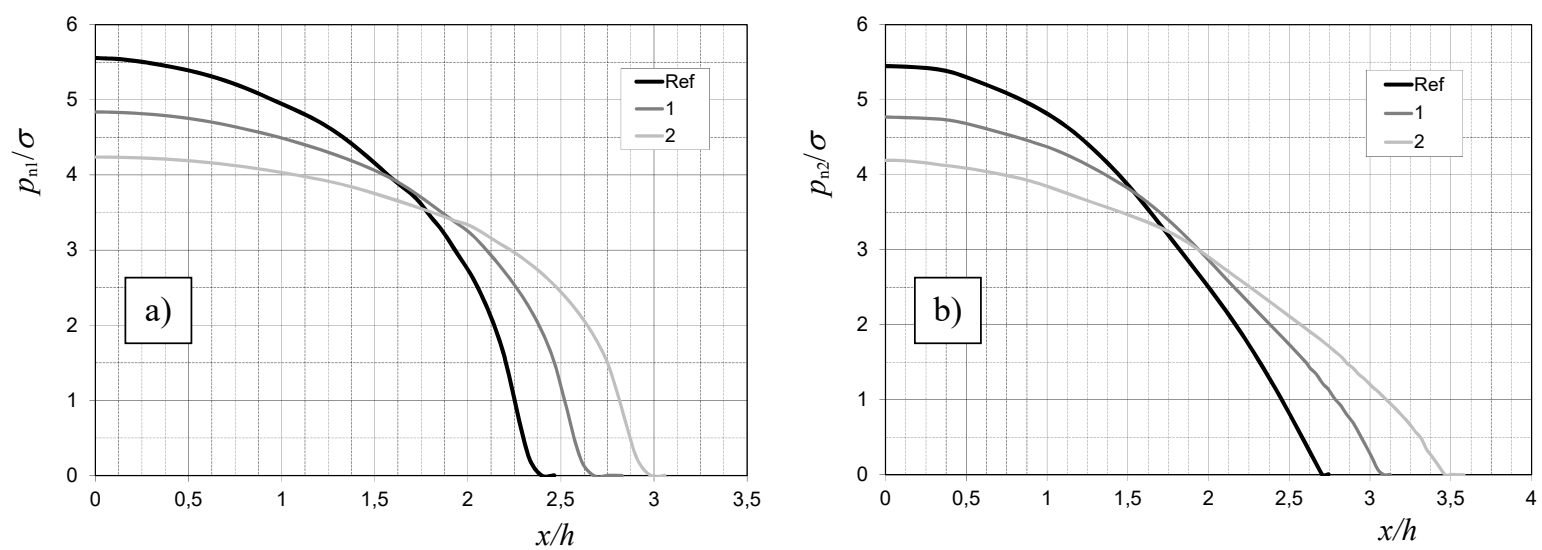

Fig. B6 Contact pressure distributions for different material properties of the substrate for $R / h=200$; a) at the indenter-layer interface, b) at the layer-substrate interface
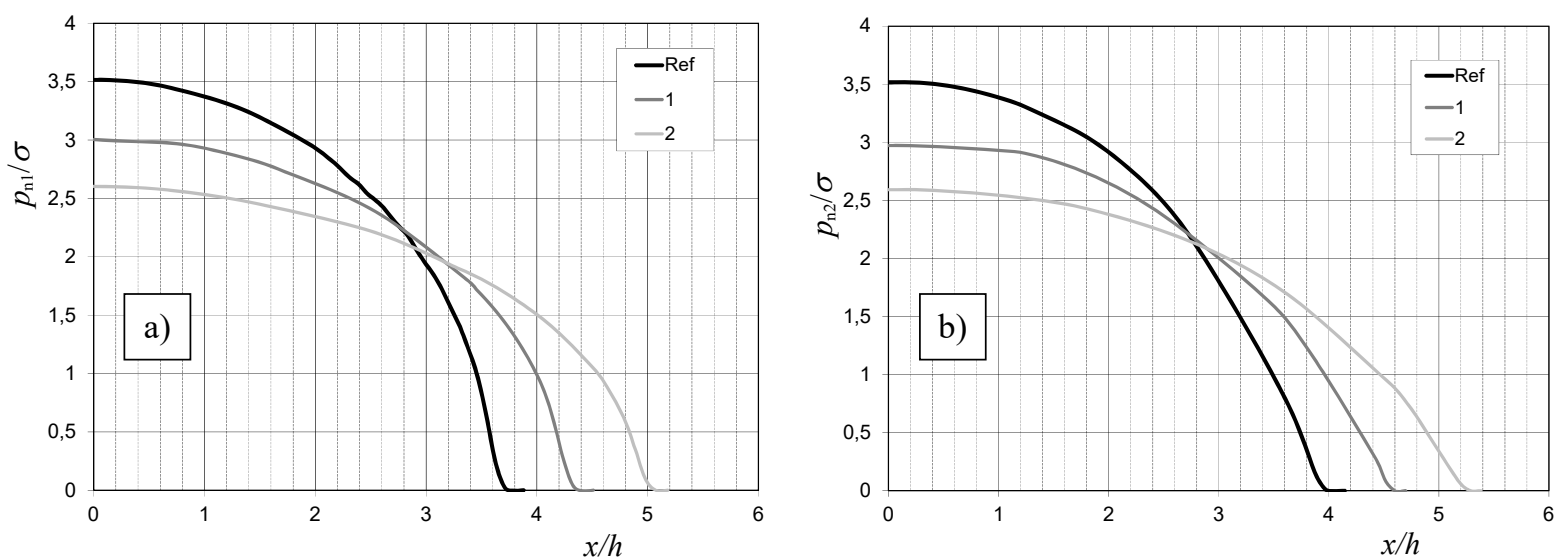

Fig. B7 Contact pressure distributions for different material properties of the indenter for $R / h=500$; a) at the indenter-layer interface, b) at the layer-substrate interface 



Fig. B8 Contact pressure distributions for different material properties of the layer for $R / h=500$; a) at the indenter-layer interface, b) at the layer-substrate interface
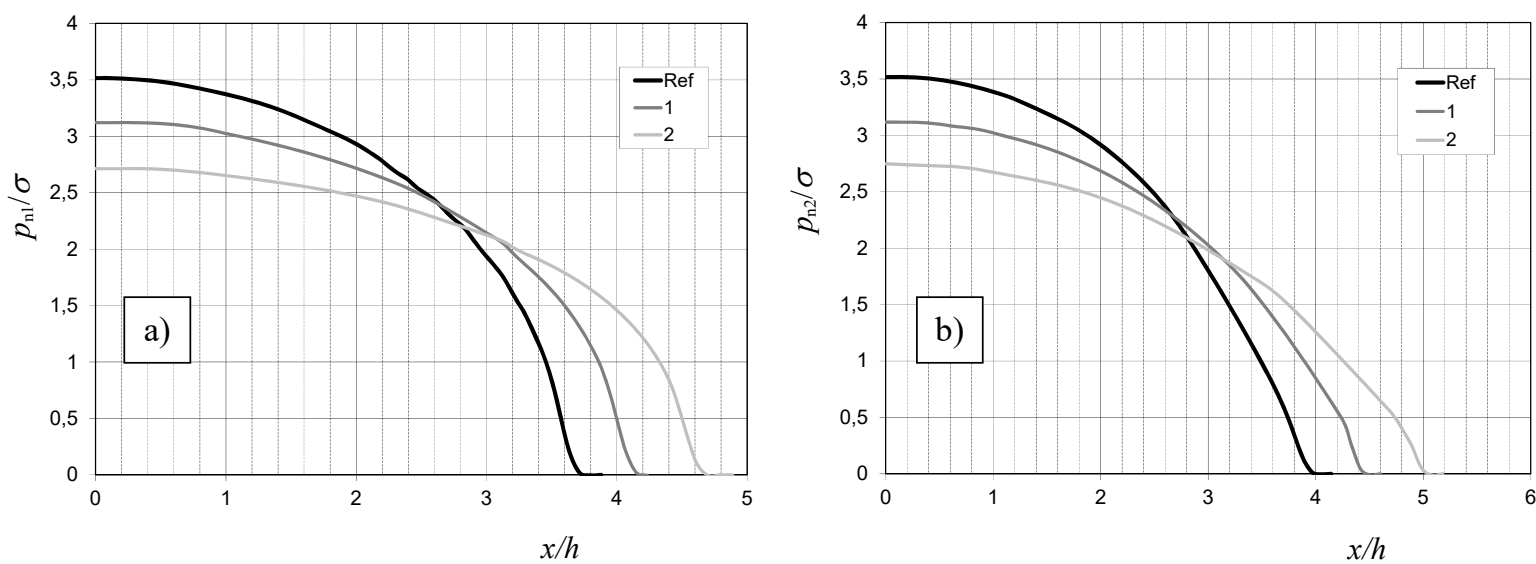

Fig. B9 Contact pressure distributions for different material properties of the substrate for $R / h=500$; a) at the indenter-layer interface, b) at the layer-substrate interface

\section{REFERENCES}

[1] K. L. Johnson: Contact mechanics, Cambridge University Press, Cambridge, 1992.

[2] L. N. G. Filon: On an Approximate Solution for the Bending of a Beam of Rectangular Cross-Section under any System of Load, with Special Reference to Points of Concentrated or Discontinuous Loading. Philosophical Transactions of the Royal Society of London, 1903, A 201, pp. 63-155.

[3] A. J. Durelli, V. J. Parks, J. S. Nørgård: Photoelastic solution of stresses in the elastic foundation supporting a plate. International Journal of Solids and Structures Vol. 9,1973, pp. 193-202. DOI: 10.1016/0020-7683(73)90097-8

[4] Y. Weitsman: On the Unbonded Contact Between Plates and an Elastic Half Space. Transactions of the ASME - Journal of Applied Mechanics Vol. 36 (4), Series E, 1969, pp. 198-202. DOI: 10.1115/1.3564607

[5] Y. Weitsman: A tensionless contact between a beam and an elastic half space. International Journal of Engineering Science Vol. 10 (1), 1972, pp. 73-81. DOI: 10.1016/0020-7225(72)90075-4

[6] L. M. Keer, J. Dundurs, K. C. Tsai: Problems Involving a Receding Contact Between a Layer and a Half Space. Transactions of the ASME - Journal of Applied Mechanics Vol. 39 (4), 1972, Series E, pp. 11151120. DOI: $10.1115 / 1.3422839$

[7] K. C. Tsai, J. Dundurs, L. M. Keer: Elastic Layer Pressed Against a Half Space. Transactions of the ASME - Journal of Applied Mechanics Vol. 41 (3), 1974, Series E, pp. 703-707. DOI: 10.1115/1.3423375

[8] G. M. L. Gladwell: On Some Unbonded Contact Problems in Plane Elasticity Theory. Transactions of the ASME - Journal of Applied Mechanics Vol. 43 (3), 1976, Series E, pp. 263-267. DOI: 10.1115/1.3423821

[9] M. Ratwani, F. Erdogan: On the plane contact problem for a frictionless elastic layer. International Journal of Solids and Structures Vol. 9 (8), 1973, pp. 921-936. DOI: 10.1016/0020-7683(73)90021-8

[10] M. B. Civelek, F. Erdogan: The axisymmetric double contact problem for a frictionless elastic layer. International Journal of Solids and Structures Vol. 10 (6), 1974, pp. 639-659.

DOI: $10.1016 / 0020-7683(74) 90048-1$ 
[11] J. A. Garrido, A. Foces, F. Paris: B.E.M. applied to receding contact problems with friction. Mathematical and Computer Modelling Vol. 15, 1991, pp. 143-153. DOI: 10.1016/0895-7177(91)90060-K

[12] J. A. Garrido, A. Lorenzana: Receding contact problem involving large displacements using the BEM. Engineering Analysis with Boundary Elements Vol. 21, 1998, pp. 295-303. DOI: 10.1016/S09557997(98)00018-6

[13] J. J. Kauzlarich, J. A. Greenwood: Contact between a centrally loaded plate and a rigid or elastic base, with application to pivoted pad bearings. Proceedings of the Institution of Mechanical Engineers, Part C: Journal of Mechanical Engineering Science Vol. 215 (6), 2001, pp. 623-628. DOI: $10.1243 / 0954406011523965$

[14] I. Comez, A.Birinci, R. Erdol: Double receding contact problem for a rigid stamp and two elastic layers. European Journal of Mechanics - A/Solids Vol. 23, 2004, pp. 301-309. DOI: 10.1016/j.euromechsol.2003.09.006

[15] V. Kahya, T. S. Ozsahin, A. Birinci, R. Erdol: A receding contact problem for an anisotropic elastic medium consisting of a layer and a half plane. International Journal of Solids and Structures Vol. 44, 2007, pp. 5695-5710. DOI: 10.1016/j.ijsolstr.2007.01.020

[16] S. El-Borgi, R. Abdelmoula, L. Keer: A receding contact plane problem between a functionally graded layer and a homogenous substrate. International Journal of Solids and Structures Vol. 43, 2006, pp. 658674. DOI: 10.1016/j.ijsolstr.2005.04.017

[17] M. Rhimi, S. El-Borgi, W. Ben Saïd, F. Ben Jemaa: A receding contact axisymmetric problem between a functionally graded layer and a homogenous substrate. International Journal of Solids and Structures Vol. 46, 2009, pp. 3633-3642. DOI: 10.1016/j.ijsolstr.2009.06.008

[18] Y. J. Ahn, J. R. Barber: Response of frictional receding contact problems to cyclic loading. International Journal of Mechanical Sciences Vol. 50, 2008, pp. 1519-1525. DOI: 10.1016/j.ijmecsci.2008.08.003

[19] I. Comez: Frictional contact problem for a rigid cylindrical stamp and an elastic layer resting on a half plane. International Journal of Solids and Structures Vol. 47, 2010, pp. 1090-1097. DOI: 10.1016/j.ijsolstr.2010.01.003

[20] H. Adibelli, I. Comez, R. Erdol: Receding contact problem for a coated layer and a half-plane loaded by a rigid cylindrical stamp. Archives of Mechanics Vol. 65 (3), 2013, pp. 219-236.

[21] E. Öner, M. Yaylaci, A. Birinci: Solution of a receding contact problem using an analytical method and a finite element method. Journal of Mechanics of Materials and Structures Vol. 9 (3), 2014, pp. 333-345. DOI: 10.2140/jomms.2014.9.333

[22] A. Birinci, G. Adiyaman, M. Yaylaci, E. Öner: Analysis of continuous and discontinuous cases of a contact problem using analytical method and FEM. Latin American Journal of Solids and Structures Vol. 12 (9), 2015, pp. 1771-1789.

[23] E. Öner, M. Yaylaci, A. Birinci: Analytical solution of a contact problem and comparison with the results from FEM. Structural Engineering and Mechanics Vol. 54 (4), 2015, pp. 607-622. DOI: $10.12989 /$ sem.2015.54.4.607

[24] R. Allahabadi: Three Dimensional Slideline Contact. MSC/NASTRAN World User's Conference, 1993.

[25] MSC.Nastran 2004 Quick Reference Guide. MSC.Software Corporation, 2003.

[26] K. Iyer: Solutions for contact in pinned connections. International Journal of Solids and Structures Vol. 38, 2001, pp. 9133-9148. DOI: 10.1016/S0020-7683(01)00054-3

[27] GOM mbH. ARAMIS User Information - Hardware. GOM mbH, 2008.

Submitted: $\quad 24.12 .2015$

Accepted: $\quad 10.5 .2016$
Branimir Rončević

Department of Mechanical Engineering Design, Faculty of Engineering,

University of Rijeka

Vukovarska 58, 51000 Rijeka, Croatia broncev@riteh.hr

Ante Bakić

INETEC - Institute for Nuclear Technology

Dolenica 28, 10250 Lučko, Croatia

Janoš Kodvanj

Department of Engineering Mechanics,

Faculty of Mechanical Engineering and

Naval Architecture, University of Zagreb

Ivana Lučića 5, 10000 Zagreb, Croatia 